\title{
Inserção da psicologia nos cursos de graduação em odontologia: contribuição na humanização do cuidado em saúde
}

\author{
- Danielle Fernandes Lopes Faculdade de Odontologia, Universidade Federal de Juiz de Fora (UFJF), Juiz de Fora, MG, \\ Brasil • Yuri de Lima Medeiros Faculdade de Odontologia, Universidade Federal de Juiz de Fora (UFJF), Juiz de Fora, \\ MG, Brasil - Luan Viana Faria Faculdade de Odontologia, Universidade Federal de Juiz de Fora (UFJF), Juiz de Fora, \\ MG, Brasil • João Paulo Santana da Silva Faculdade de Odontologia, Universidade Federal de Juiz de Fora (UFJF), \\ Juiz de Fora, MG, Brasil • Fabiane Rossi dos Santos Grincenkov Departamento de Psicologia da Universidade \\ Federal de Juiz de Fora (UFJF), Juiz de Fora, MG, Brasil • Gracieli Prado Elias Departamento de Odontologia Social e \\ Infantil da Universidade Federal de Juiz de Fora (UFJF), Juiz de Fora, MG, Brasil
}

RESUMO || Objetivo: Com base na notória necessidade da presença da disciplina de psicologia nas grades curriculares do ensino superior de cursos de saúde, buscou-se compreender qual sua oferta nas graduações em odontologia, obtendo-se um panorama da inserção da disciplina de psicologia nos projetos pedagógicos dos cursos de graduação em odontologia da região Sudeste do Brasil. Método: Realizou-se um estudo transversal no qual as grades curriculares dos cursos foram analisadas, buscando-se as informações referentes às variáveis: oferta da disciplina, natureza do componente curricular (obrigatória ou não-obrigatória), forma em que o conteúdo é ministrado (presencial ou à distância) e carga horária. Resultados: Participaram do estudo 176 faculdades, das quais 32 foram excluídas por não disponibilizarem suas respectivas grades curriculares. Observou-se que 98 (68,05\%) instituições de ensino superior (IES) oferecem a disciplina de em sua grade curricular, sendo 78,44\% obrigatória, 78,57\% presencial e com carga horária máxima de 80 e mínima de 3oh. Conclusão: Conclui-se que, embora seja ofertada na maioria das IES, é necessária a elevação da inclusão dessa disciplina em toda a amostra estudada, visto que ela é de grande relevância para a humanização do atendimento odontológico.

ABSTRACT | Inclusion of psychology in undergraduate dentistry courses: contribution to a humanized healthcare - Objective: Based on the evident need for classes on psychology in higher education curricula, we sought to understand its offer in undergraduate Dentistry courses in southeast Brazil, thus obtaining an overview on how undergraduate pedagogical projects in Dentistry include the discipline of Psychology. Method: This cross-sectional study analyzed the undergraduate programs, collecting the following variables: if the discipline is offered, nature of the curricular component (mandatory or elective), format (in-person or distance learning) and course load. Results: Our sample consisted of 176 faculties, of which we excluded 32 for not providing their respective curriculum. Results show that 98 (68.05\%) higher education institutions (HEIs) offer a class on Psychology in their curriculum, with 78.44\% of them being mandatory, $78.57 \%$ in-person, with maximum course load of 80 hours and minimum of 30 hours. Conclusion: Although the discipline of Psychology is offered by most HEIs, its inclusion should reach all institutions analyzed, since the field is of great relevance for humanizing dental care.

DESCRIPTORS | Behavioral Medicine; Humanization of Assistance; Education Dental; Curriculum.

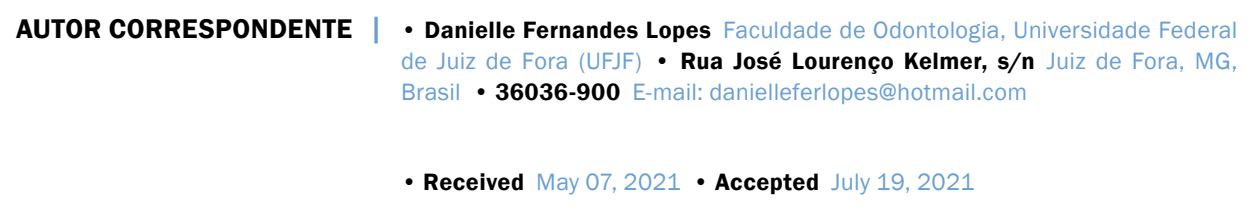

• Dol: http://dx.doi.org/10.11606/issn.2357-8041.clrd.2021.185307 


\section{INTRODUÇÃO}

A prática odontológica geralmente é associada a episódios de ansiedade, de estresse e, até mesmo, de fobia. ${ }^{1}$ Essa situação é agravada especialmente em casos que envolvem anestesia local e procedimentos cirúrgicos. ${ }^{2}$ Pacientes com alterações sistêmicas, principalmente desordens cardiovasculares, podem ter seus sinais vitais alterados frente às situações de nervosismo, levando a complicações transoperatórias no atendimento odontológico, como o aumento do risco hemorrágico, a exacerbação da dor e a necessidade de maiores quantidades de anestésico local. ${ }^{3}$

Diante desse contexto e frente ao aumento crescente da integração entre as áreas da saúde, a psicologia se destaca na intervenção em hábitos deletérios e nos cuidados em saúde bucal, com foco no manejo dos pacientes odontológicos. ${ }^{4}$ Modelos e teorias psicológicas, como a entrevista motivacional e a intervenção social cognitiva, estão sendo utilizados em ações de conscientização sobre a saúde bucal, apresentando melhor eficácia quando comparada à educação em saúde tradicional. ${ }^{5}$ Além disso, a psicologia aplicada, em suas diferentes nuances, tem sido preferencialmente adotada em odontologia, optando-se pelas técnicas de manejo comportamentais, como a musicoterapia, a distração e a hipnose, uma vez que outras formas de abordagem, como o uso de medicamentos sedativos para redução da ansiedade, têm sido utilizadas de forma mais limitada, devido aos efeitos colaterais associados. $^{6}$

Paralelo a isso, as diretrizes nacionais curriculares (DNC) do curso de odontologia, revisadas e aprovadas em 2018,7 determinam que a formação do cirurgião-dentista (CD) deve contemplar não só as ciências odontológicas, mas também as dimensões humanísticas e sociais no atendimento dos pacientes, o que acontece por meio da disciplina de psicologia aplicada à odontologia. Assim, o CD deve utilizar das bases referenciais da psicologia na relação profissional-paciente durante o tratamento das diferentes faixas etárias, contribuindo, dessa forma, na compreensão dos seus determinantes sociais, psicológicos e comportamentais, a nível individual e coletivo.

No entanto, essas diretrizes não especificam os aspectos referentes à carga horária e à ementa da disciplina de psicologia aplicada à odontologia. Esta é descrita como uma disciplina multidisciplinar de conhecimentos teóricos, obtidos através da psicologia da saúde e aplicados às atividades de avaliação, controle e modificação de hábitos e comportamentos de pacientes expostos aos cuidados de saúde bucal. Assim, sua principal finalidade é a intervenção dos aspectos psicossociais durante os procedimentos odontológicos, buscando a promoção do bem-estar e da qualidade de vida dos indivíduos, bem como a facilitação do enfrentamento de situações de agravo dos transtornos bucais. ${ }^{8}$

O currículo mínimo de Odontologia passou a incluir a disciplina de psicologia desde a Resolução $\mathrm{n}^{\mathrm{0}}$ 04/82 do Conselho Federal de Educação (CFE). ${ }^{9}$ Contudo, não há na literatura nenhum estudo de verificação da inclusão dessa disciplina nas grades curriculares das instituições de ensino superior (IES) que ofertam o curso.

Sendo assim, o presente estudo tem o objetivo de verificar a inclusão da disciplina de psicologia nos projetos políticos pedagógicos dos cursos de odontologia da região Sudeste do Brasil, escolhida por ser a região onde há maior concentração dos cursos de odontologia no país. ${ }^{10}$

\section{METODOLOGIA Cenárío}

A resolução $\mathrm{n}^{0}$ 04/82 do $\mathrm{CFE}^{9}$ solicita que a presença da disciplina de psicologia seja parte do currículo mínimo do curso de odontologia, de modo a favorecer o cumprimento da proposta das diretrizes curriculares nacionais.7 Esta necessidade parte da premissa de que a disciplina auxilia para uma formação mais humanizada e integral do aluno. 


\section{Desenho de estudo}

Estudo de escopo descritivo, transversal e exploratório com abordagem quantitativa para oferecer um panorama a respeito do preparo de cirurgiões-dentistas durante a graduação para lidar com situações de cunho psicológico que envolvam o atendimento odontológico.

\section{Procedimentos}

Foi realizada uma pesquisa acerca de todas as instituições de ensino superior públicas e privadas que ofertavam o curso de odontologia cadastradas no portal eletrônico e-MEC até fevereiro de 2019. Esse portal é regulamentado pela Portaria Normativa $\mathrm{n}^{0} 21$, de 21 de dezembro de 2017 e pode ser acessado através do portal www.emec.mec.gov.br. ${ }^{11}$

Em seguida, foi executada uma busca referente às IES que ofereciam a disciplina de "Psicologia" ou "Psicologia aplicada à Odontologia" em suas grades curriculares por meio do seu sítio eletrônico.

A coleta de dados foi realizada entre os meses de março e junho de 2019. Àquelas que não disponibilizaram, foi enviado um e-mail para a coordenação do curso, solicitando o material, com o prazo de dois meses para que enviasse uma resposta. Foram analisados todos os sítios desses cursos, buscando as seguintes informações sobre a disciplina de psicologia em suas respectivas grades curriculares: (i) categoria administrativa da IES (pública ou privada), (ii) inserção e oferta da disciplina, (iii) natureza do componente curricular (obrigatória ou não-obrigatória), (iv) ensino presencial ou à distância e (v) carga horária total.

\section{Critérios de seleção}

Foram elegíveis para participar do estudo as IES da região sudeste que disponibilizaram o acesso a sua grade curricular no período determinado por e-mail ou através de sua plataforma online. Das 176 instituições cadastradas, foram incluídas no estudo 144, obedecendo aos critérios adotados pela equipe de pesquisa.

\section{Aspectos éticos}

O estudo utilizou consulta em arquivos de instituições, que constituem dados de domínio público, e não houve envolvimento de seres humanos ou animais. Desta forma, dispensa-se a submissão para apreciação do Comitê de Ética em Pesquisa (CEP).

\section{Análise de dados}

Os dados foram analisados e tabulados com o programa GraphPad Prism 8.1.2 (GraphPad Software Inc., La Jolla, CA, EUA) e por meio de estatísticas descritivas.

\section{RESULTADOS}

A distribuição das 144 IES incluídas no estudo, de acordo com sua localização geográfica, pode ser descrita da seguinte forma: Espírito Santo (ES, $\mathrm{n}=12$ ), Minas Gerais (MG, $\mathrm{n}=61$ ), Rio de Janeiro $(\mathrm{RJ}, \mathrm{n}=28$ ) e São Paulo (SP, $\mathrm{n}=75$ ).

Percebe-se que, das IES cadastradas no Portal do MEC e que disponibilizam sua grade curricular, a maioria oferta a disciplina de psicologia no curso de odontologia (68,5\%).

Ao analisar a distribuição por estados, nota-se que o estado de Minas Gerais apresentou a maior porcentagem de instituições que ministram a disciplina (82,35\%), seguido pelo Espírito Santo (70\%).

Quando ofertada, a disciplina é predominantemente obrigatória (71,5\% no ES, 83,3\% em MG, 83,4\% no RJ e 56,8\% em SP) e quanto à metodologia de ensino, pode-se observar uma variação descrita com presencial (88,7\%), à distância (4,1\%) e semipresencial $(3,1 \%)$, com a primeira modalidade sendo a mais observada em todos os estados. Todos estes dados encontram-se explicitados e detalhados na Tabela 1. 
Tabela 1 | Descrição dos dados encontrados, para as variáveis em estudo, por estados da região Sudeste.

\begin{tabular}{|c|c|c|c|c|c|}
\hline & ES & MG & RJ & SP & Total \\
\hline Presença da disciplina & $7(70 \%)$ & $42(82,35 \%)$ & $12(57,14 \%)$ & $37(59,67 \%)$ & $98(68,05 \%)$ \\
\hline \multicolumn{6}{|l|}{ Caráter da disciplina } \\
\hline Optativa & $2(28,5 \%)$ & $6(14,3 \%)$ & $1(8,3 \%)$ & $8(21,6 \%)$ & $17(17,3 \%)$ \\
\hline Obrigatória & $5(71,5 \%)$ & $35(83,3 \%)$ & $10(83,4 \%)$ & $21(56,8 \%)$ & $71(72,4 \%)$ \\
\hline Não informado & $0(0 \%)$ & $1(2,4 \%)$ & $1(8,3 \%)$ & $8(21,6 \%)$ & $10(10,3 \%)$ \\
\hline \multicolumn{6}{|l|}{ Modalidade de ensino } \\
\hline Presencial & $4(57,2 \%)$ & $37(88,1 \%)$ & $11(91,7 \%)$ & $35(94,6 \%)$ & $87(88,7 \%)$ \\
\hline À distância & - & $3(7,1 \%)$ & - & $1(2,7 \%)$ & $4(4,1 \%)$ \\
\hline Semipresencial & $2(28,5 \%)$ & $1(2,4 \%)$ & - & - & $3(3,1 \%)$ \\
\hline Não identificado & $1(14,3 \%)$ & $1(2,4 \%)$ & $1(8,3 \%)$ & $1(2,7 \%)$ & $4(4,1 \%)$ \\
\hline
\end{tabular}

No tocante à carga horária da disciplina, quando ela é oferecida, observamos que em todos os estados a carga horária máxima observada foi de 80 horas e a mínima de 30 horas. Quanto às médias das cargas horárias, o Espírito Santo apresenta como média 72,8 horas, sendo a maior de todas, seguido por São Paulo, com 53,79 horas. Esta distribuição se encontra descrita minuciosamente no Gráfico 1.

Gráfico 1 | Distribuição da carga horária da disciplina, o máximo, mínimo e a média de cada estado. Foram disponibilizadas as cargas horárias de 7 IES do Espírito Santo, 32.

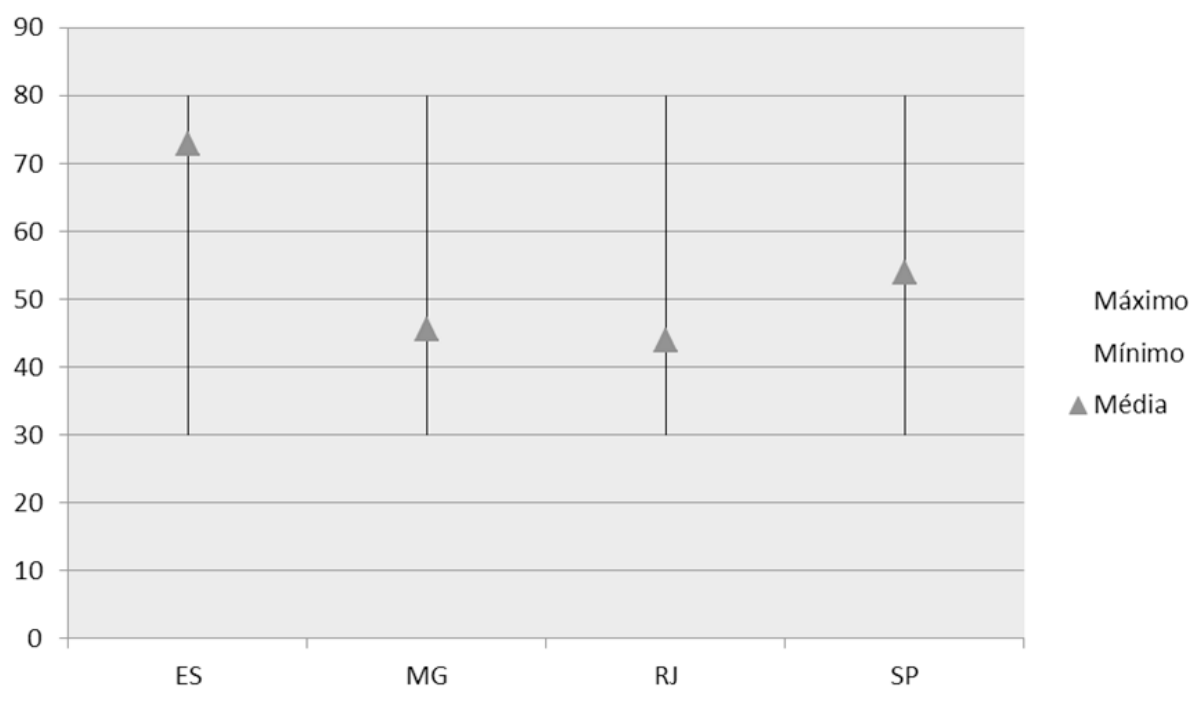

\section{DISCUSSÃO}

O Sistema Único de Saúde (SUS) tem como um dos seus princípios o cuidado integral do indivíduo nas diferentes etapas da vida, o que vai ao encontro das diretrizes curriculares dos cursos de saúde, que têm como um de seus objetivos garantir a capacitação dos alunos para a integralidade e a humanização dos atendimentos prestados à comunidade. ${ }^{712}$

Como disciplina, o conteúdo trabalhado pode ser abordado de maneira mais ampla e de modo 
mais efetivo e produtivo, o que culmina com o melhor aproveitamento do discente de odontologia. Os conceitos relacionados à humanização e à integralidade no atendimento, seguindo o novo conceito da Organização Mundial da Saúde ${ }^{13}$ no que tange à saúde como um "estado de completo bemestar físico, mental e social e não somente ausência de afecções e enfermidade", podem ser estudados como parte integrante de toda profissão e não apenas abordados como tópicos de uma ou mais especialidades isoladas.

Assim, por meio da avaliação das matrizes curriculares das 144 IES abordadas nesse estudo, observa-se uma divergência frente ao que é recomendado pelas diretrizes nacionais curriculares, uma vez que somente $68,05 \%$ delas apresentam cursos de odontologia que oferecem a disciplina de psicologia, muito embora, na maioria das vezes, a psicologia apareça como disciplina de caráter obrigatório $(72,4 \%)$ e seja ministrada de forma presencial $(88,7 \%)$. No entanto, tal fato é alarmante, em virtude de que a ausência desses conhecimentos pode prejudicar a formação e a capacitação do discente em odontologia, haja vista que a formação social, humanística e ética deve ser adquirida através do oferecimento de disciplinas de cunho social. ${ }^{14,15}$

Além disso, a ausência da disciplina prejudica o debate de conceitos importantes, como os determinantes sociais em saúde, contrapondo a realidade atual que busca fomentar discussões a este respeito por meio da criação da Comissão Nacional sobre os Determinantes Sociais da Saúde (CNDSS). O não conhecimento deste discurso gera dificuldades no atendimento integral e danifica a humanização em saúde, uma vez que a falta de compreensão da realidade que o paciente está inserido pode prejudicar a abordagem e a terapêutica que ele necessita. ${ }^{16}$

O conhecimento da psicologia na área odontológica também é positivo no preparo do graduando para o uso de Práticas Integrativas e
Complementares de Saúde (PIC) no alívio da dor e controle da ansiedade. $\mathrm{O}$ uso de plantas medicinais como terapia para a ansiedade e a homeopatia para tratamento de disfunções têm sido cada vez mais relatados na literatura, ${ }^{17-19}$ bem como a aplicabilidade da acupuntura para redução de dores em pacientes bruxistas e no pós-operatório de cirurgias orais..$^{20,21}$ Importante ressaltar que todas essas terapias fazem parte da Política Nacional de Práticas Integrativas e Complementares (PNPIC) e foram institucionalizadas no Sistema Único de Saúde (SUS). Elas apresentam em comum a visão integral do indivíduo, considerando, no tratamento e na abordagem, seu perfil físico e emocional, com vistas à humanização do atendimento, à escuta acolhedora, ao desenvolvimento do vínculo terapêutico e à integração do ser humano com o meio ambiente e a sociedade. ${ }^{22}$

Considerando-se que o aluno de graduação não tenha contato com as novas realidades do sistema de saúde brasileiro e do que se encontra disponível para o atendimento com abordagem integral ao paciente, é possível aventar que a falta de informações a respeito da eficácia e da atuação dessas práticas corrobore com o estigma dos preceitos alopáticos, aumentando o preconceito com a prática desses tratamentos, relacionando-os a questões simbólicas e sem embasamento científico. ${ }^{23}$ Deste modo, as IES não estarão preparando adequadamente o futuro profissional para o mercado de trabalho e, consequentemente, para a atuação no sistema de saúde brasileiro, o que também é uma das atuais diretrizes curriculares dos cursos de odontologia no Brasil.?

A falta do conteúdo de psicologia nos programas pedagógicos é preocupante, uma vez que pode justificar possíveis dificuldades que cirurgiõesdentistas têm em lidar, por exemplo, com a ansiedade dos pacientes. Experiências odontológicas desagradáveis e de sofrimento podem prejudicar tratamentos odontológicos posteriores, haja vista 
que este é um dos fatores que favorecem o quadro de ansiedade. ${ }^{24}$ Além disso, o processo de adoecimento e recuperação partem de uma série de fatores biológicos e psicossociais que tangenciam o estado do paciente, do profissional e do contexto em que eles se encontram. ${ }^{25}$

Além do conteúdo, o que se pode observar no atual estudo é que não houve concordância em relação à carga horária fornecida à disciplina, nem em relação a sua adoção em caráter obrigatório, o que sugere a necessidade de uma padronização pelos órgãos competentes, visando um melhor aproveitamento e preparo dos discentes, o que certamente refletirá no atendimento ao paciente.

É necessário repensar as práticas formativas em saúde de modo a atender as necessidades da população, em busca de satisfazer sua demanda e repensar formas de tratamento que atuem de forma humana e integral no processo saúde-doençacuidado. ${ }^{26}$ Para isso, é ideal que este debate circule dentro das IES, atendendo ao que se pede nas DNC e na legislação vigente, principalmente nas disciplinas de conteúdo embasado nas ciências humanas e na antropologia, como a psicologia.

Importante ressaltar ainda que um dos fatores limitantes para a realização desse estudo foi a falta de informações disponibilizadas pelas instituições nas grades curriculares, conforme apontam também outros estudos com metodologias semelhantes, os quais são de extrema relevância para o aperfeiçoamento do ensino de excelência. ${ }^{15,27-34}$ Assim sendo, o número de IES que apresentam a disciplina de psicologia em sua grade curricular pode ser um pouco maior do que o mostrado no estudo atual, uma vez que 32 IES tiveram que ser excluídas da amostra.

\section{CONCLUSÃO}

O presente estudo possibilitou traçar um panorama da inserção da disciplina de psicologia nas grades curriculares dos cursos de odontologia da região Sudeste do Brasil. Embora ela seja ofertada na maioria das IES (68\%), acredita-se que o aumento de sua inclusão como disciplina independente nos cursos de odontologia favorecerá a formação dos discentes, uma vez que seu conteúdo deve ser abordado de maneira ampla, contemplando o curso como um todo, com vistas à humanização no atendimento e à formação qualificada, obedecendo as atuais diretrizes curriculares do curso.

\section{REFERÊNCIAS}

1. De Stefano R. Psychological Factors in Dental Patient Care: Odontophobia. Medicina (Kaunas). 2019;55(10):2-4. doi: 10.3390/medicina55100678.

2. Sirin Y, Humphris GM, Sencan S, Firat D. What is the most fearful intervention in ambulatory oral surgery? Analysis of an outpatient clinic. Int J Oral Maxillofac Surg. 2012;41(10):1284-90. doi: 10.1016/j.ijom.2012.06.013.

3. Migliau G. Dental management of patients with cardiovascular disease. Minerva Stomatol. 2002;51(1-2):49-63.

4. Murrer RD, Francisco SS. Diagnóstico e Manejo da Ansiedade Odontológica pelos Cirurgiões-Dentistas. Interacao Psicol. 2015;19(1):37-46. doi: 10.5380/psi.v19i1.35561.

5. Werner H, Hakeberg M, Dahlström L, Eriksson M, Strandell A, Svanberg T, et al. Psychological Interventions for Poor Oral Health: A Systematic Review. J Dent Res. 2016;95(5):506-14. doi: 10.1177/0022034516628506.

6. Burghardt S, Koranyi S, Magnucki G, Strauss B, Rosendahl J. Non-pharmacological interventions for reducing mental distress in patients undergoing dental procedures: Systematic review and meta-analysis. J Dent. 2018;69:22-31. doi: 10.1016/j.jdent.2017.11.005.

7. Conselho Nacional de Educação (BR), Câmara de Educação Superior (BR). Parecer CNE/CES n. ${ }^{\circ}$ 803/2018, de 5 de dezembro de 2018. Diretrizes Curriculares Nacionais do Curso de Graduação em Odontologia. Diário Oficial da União. 5 dez 2018.

8. Moraes ABA, Pessotti I. Psicologia aplicada à odontologia. São Paulo: Sarvier; 1985.

9. Conselho Federal de Educação (BR). Resolução n. ${ }^{\circ}$ 04/82. Diário Oficial da União. 1982.

10. Martin ASS, Chisini LA, Martelli S, Sartori LRM, Ramos EC, Demarco FF. Distribuição dos cursos de Odontologia e de cirurgiões-dentistas no Brasil: uma visão do mercado de trabalho. Rev ABENO. 2018;18(1):63-73. doi: 10.30979/rev. abeno.v18i1.399 
11. Ministério da Educação (BR). Cadastro e-MEC de Instituições e Cursos de Educação Superior [Internet]. Brasília; 2017 [citado 22 fev 2019]. Disponível em: www.emec.mec.gov.br.

12. Conselho Nacional de Educação (BR), Câmara de Educação Superior (BR). Parecer CES/CNE n. ${ }^{0}$ 1.300/2001, de março de 2002. Diretrizes Curriculares Nacionais para os Cursos de Graduação em Farmácia e Odontologia. Diário Oficial da União. 4 mar 2002.

13. Organización Mundial de la Salud. Documentos básicos. 26 ed. Ginebra: OMS; 1976.

14. Ministério da Saúde (BR), Ministério da Educação (BR). A trajetória dos cursos de graduação na saúde de 1991-2004. Brasília: Inep; 2006.

15. Medeiros YL, Lopes DF, Faria LV, Soares MRPS, Silvério CCP. Ensino da Língua Brasileira de Sinais nos cursos de graduação em Odontologia do Sudeste brasileiro: um estudo transversal. Rev ABENO. 2020;20(1):113-20. doi: 10.30979/ rev.abeno.v2oi1.933

16. Buss PM, Pellegrini Filho A. A saúde e seus determinantes sociais. Physis. 2007;17(1):77-93. doi: 10.1590/So10373312007000100006

17. Baatsch B, Zimmer S, Recchia DR, Büssing A. Complementary and Alternative Therapies in Dentistry and Characteristics of Dentists Who Recommend Them. Complement Ther Med. 2017;35:64-9.

18. Martínez CC, Gómez MD, Oh MS. Use of Traditional Herbal Medicine as an Alternative in Dental Treatment in Mexican Dentistry: A Review. Pharm Biol. 2017;55(1):1992-8. doi: 10.1080/13880209.2017.1347188.

19. Silva SR. Incorporando novas ferramentas. Rev Assoc Paul Cir Dent. 2001;55(4):233-42.

20. Dallanora LJ, Feltrin PP, Inoue RT, Santos VA, Tanaka J. Avaliação no uso de acupuntura no tratamento de pacientes com bruxismo. Rev Gaucha Odontol. 2004;52(5):333-9.

21. Sertel S, Herrmann S, Greten HJ, Haxsen V, El-Bitar S, Simon $\mathrm{CH}$, et al. Additional use of acupuncture to NSAID effectively reduces post-tonsillectomy pain. Eur Arch Otorhinolaryngol. 2009;266(6):919-25. doi: 10.1007/s00405-008-0851-1.

22. Ministério da Saúde (BR). Política Nacional de Práticas Integrativas e Complementares no SUS - PNPIC-SUS. Brasília; 2005.

23. Assis WC, Britto FR, Vieira LO, Santos ES, Boery RNSO, Duarte ACS. Novas formas de cuidado através das práticas integrativas no sistema único de saúde. Rev Bras Promoc Saude. 2018;31(2):1-6. doi: 10.5020/18061230.2018.7575.
24. Scandiuzzi-Francisco S, Souza H, Barros-Neto A, Hildebrando A, Chaves K, Murrer R, et al. Avaliação do status de ansiedade durante o atendimento odontológico. Rev Cubana Estomatol. 2019;56(1):33-41.

25. DiMatteo MR, DiNicola DD. Achieving Patient Compliance: The Psychology of the Medical Practitioner's Role. New York: Pergamon; 1982.

26. Pitombeira DF, Xavier AS, Barroso REC, Oliveira PRS. Psicologia e a Formação para a Saúde: Experiências Formativas e Transformações Curriculares em Debate. Psicol Cinc Prof. 2016;36(2):280-91. doi: 10.1590/1982-3703001722014.

27. Faria LV, Oliveira GA, Grázzia MEP, Medeiros YL, Lopes DF, Leite ICG. O ensino de implantodontia nas graduações brasileiras de Odontologia: um estudo transversal. Rev Eletron Acervo Saude. 2020;12(4):e2672. doi: 10.25248/reas.e2672.2020

28. Lopes DF, Medeiros YL, Faria LV, Senra MRP. Odontologia para pacientes com necessidades especiais: como é a oferta dessa disciplina nas faculdades de odontologia do sudeste brasileiro? Arq Odontol. 2021;57:e03. doi: 10.7308/aodontol/2021.57.eo3

29. Faria LV, Medeiros YL, Lopes DF, Vilela EM, Assis NMSP. Medical emergencies teaching in dentistry undergraduate courses in southeastern Brazil. Braz J Oral Sci. 2020;19:e209247. doi: 10.20396/bjos.v19io.8659247

30. Faria LV, Medeiros YL, Lopes DF, Durso BC. DTM e dor orofacial: perspectivas curriculares das faculdades de Odontologia do Sudeste brasileiro. Hu Rev. 2020;46:1-7. doi: 10.34019/1982-8047.2020.v46.30348

31. Medeiros YL, Faria LV, Lopes DF, Oliveira IS, Fabri GMC. Inserção da Odontologia Hospitalar na grade curricular dos cursos de Odontologia do sudeste brasileiro. Rev Fac Odontol Porto Alegre. 2020;61(1):87-93. doi: 10.22456/2177-0018.101594

32. Medeiros YL, Faria LV, Lopes DF, Vilela EM. Prótese Bucomaxilofacial na educação superior em Odontologia: perspectivas curriculares. Rev Cir Traumatol Buco-Maxilo-Fac. 2020;20(4):6-11.

33. Faria LV, Pinheiro FA, Lopes DF, Medeiros YL, Silva RHA. Ensino da Odontologia Legal nos cursos de graduação em Odontologia: um estudo transversal da região sudeste brasileira. Rev Bras Odontol Leg. 2021;8(1):13-22. doi: 10.21117/rbol-v8n12021-304

34. Medeiros YL, Silva PVR, Lopes DF, Faria LV, Guimarães LDA. Oferta da disciplina de Estomatologia nos cursos de Odontologia do sudeste brasileiro. RFO UPF. 2020;25(1):26-31. doi: 10.5335/rfo.v25i1.10485 